\title{
]jfis
}

\section{Analysis of Balance of Quadrupedal Robotic Walk using Measure of Balance Margin}

\section{Byoung-Ho Kim}

Biomimetics and Intelligent Robotics Lab.

Department of Mechatronics Engineering, Kyungsung University, Busan 608-736, Korea

\begin{abstract}
In this study, we analyze the balance of quadruped walking robots. For this purpose, a simplified polygonal model of a quadruped walking configuration is considered. A boundaryrange-based balance margin is used for determining the system stability of the polygonal walking configuration considered herein. The balance margin enables the estimation of the walking configuration's balance for effective walking. The usefulness of the balance margin is demonstrated through exemplary simulations. Furthermore, balance compensation by means of foot stepping is addressed.
\end{abstract}

Keywords: Quadruped robotic walking, Balance margin, Balance compensation, Foot stepping

\section{Introduction}

Given their ability to walk, leg-based mobile manipulation systems are very convenient to work with in normal as well as in irregular environments [1-3]. For achieving the desired mobility, it is important to design a multiple-leg mechanism and manage system balance in a manner wherein there is adequate coordination among the legs. For instance, if any of the legs in a quadruped walking configuration is incompatible with the others, the robot may

Received: Jan. 3, 2013

Revised : Jun. 6, 2013

Accepted: Jun. 12, 2013

Correspondence to: Byoung-Ho Kim (kimbh@ks.ac.kr)

(C)The Korean Institute of Intelligent Systems

(c) This is an Open Access article distributed under the terms of the Creative Commons Attribution Non-Commercial License (http://creativecommons.org/licenses/ by-nc/3.0// which permits unrestricted noncommercial use, distribution, and reproduction in any medium, provided the original work is properly cited. not remain in equilibrium. Therefore, proper coordination among the legs is required for achieving system balance and the desired walking performance in the stationary state as well as while maneuvering. A performance measure is helpful for identifying system balance during robot walking. From a geometric viewpoint, a few criteria have been put forth for ensuring stable quadruped walking [4-7]. In addition, an energy-based stability margin has been considered for the potential stability of legged walking robots $[8,9]$. Recently, Bretl and Lall [10] attempted to evaluate the static equilibrium by considering the foot placement. However, the body's falling direction may not have been considered in the aforementioned approaches. In contrast, when a robot is off balance, suitable foot stepping or walking steps can be executed for restoring the system balance. Additionally, for taking a step, the robot needs to know the location of the foot to be moved. In fact, the next foot location for effective balance compensation could be determined by testing an effective measure. However, comprehensive studies on identifying and compensating for the balance of quadruped walking systems are rare.

The objective of this study is to analyze the balance of quadruped walking configurations using a boundary-range-based balance margin. In addition, suitable compensation for system balance through foot stepping is addressed. 


\section{Quadruped Walking Configuration Model}

Consider a representative quadruped robot system such as a dog, shown in Figure 1 1 . The AIBO robot is a well-known example of four-legged mobile manipulation systems. Thus, many researchers have been interested in the locomotion of the robot that can be used as a test bed for walking algorithms. The four-legged robot shown in Figure $1 \mathrm{p}$ is well known and is actually used for delivery and exploration tasks in rough terrain. A stable walking strategy is usually required for quadruped tasks, and the walking mechanism's stability is closely related to the shape of the polygon formed by its supporting feet. That is, system balance is influenced by the placement of the mechanism's feet. If the walking configuration is well balanced, the given task is executed with ease.

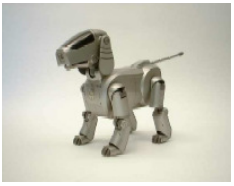

(a) AIBO [2]

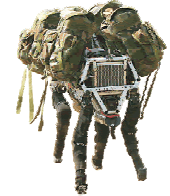

(b) BigDog [3]
Figure 1. Representative four-legged robots.

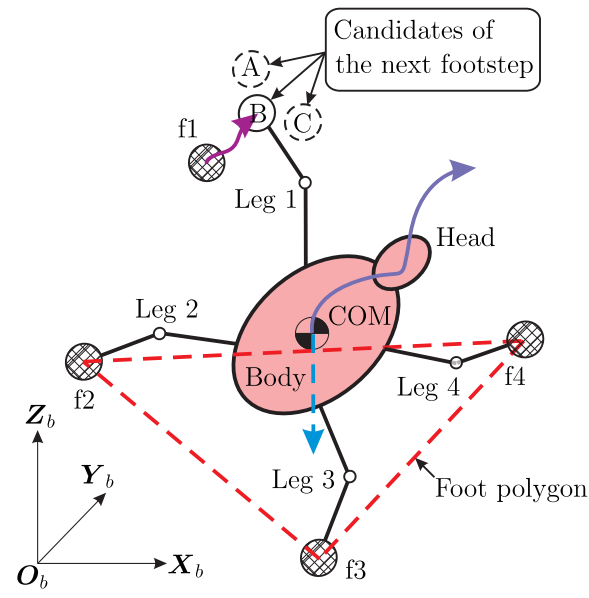

Figure 2. Polygonal model of quadruped walking configuration.

For dealing with such a quadruped walking problem, we consider a simple model of a four-legged robot, shown in Figure 2. where $X_{b}, Y_{b}, Z_{b}$ denote the $x$-, $y$-, and $z$-directional inertial frames, respectively, of the robot system. In practice, a manipulator can be attached onto the body platform for mobile manipulation tasks. However, here, we assume that the robot body includes such an interfacing manipulator. Furthermore, the freedom of the leg mechanism is adequate for the three-dimensional motion considered in this study.

So long as the vertical projection of a multi-legged robot's center of mass (COM) lies inside the supporting foot polygon [1], it can walk stably. During walking, the foot polygon varies based on the location (A, B, or C for $\mathrm{f} 1$ ) of the moving foot, as shown in Figure 2 If the location of the first foot (f1) is assigned as A, the system balance improves intuitively. Therefore, our goal is to devise a feasible measure for identifying the degree of balance of any quadruped walking configuration and demonstrate the applicability of said measure to the analysis of quadruped robotic walking.

\section{Boundary-Range-Based Balance Margin}

Consider an arbitrary motion of a quadruped robot in a landing situation, as shown in Figure 3 The landing situation implies that all feet are stably on ground, and the entire range of body motion within the operating range of the robot system is available. For effective description, we approximated body motion to the movement of the robot system's COM.

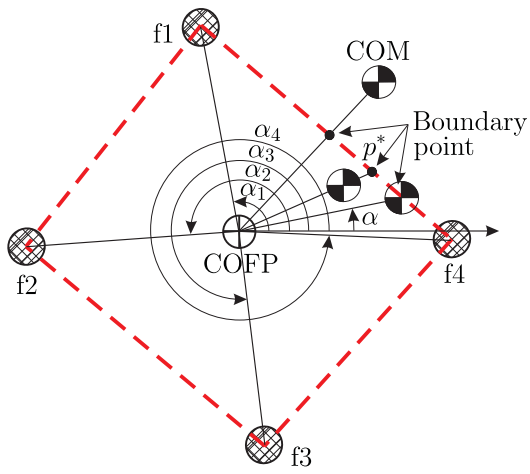

Figure 3. Boundary range and balance margin of four-legged robots. COM, center of mass; COFP, centroid of the foot polygon.

In general, a quadruped robot system can attain a balanced posture so long as the vertical projection of its $\mathrm{COM}$ lies within the boundary of its foot polygon. The balance worsens if the vertical projection of the robot system's COM lies outside the foot polygon. Based on this concept, we propose a boundaryrange-based balance margin that can be used for checking the degree of balance of a quadruped walking configuration. Figure 3 shows a schematic diagram of the process of defining the boundary range based on a quadruped robot's motion. Here, each foot lands on the $x y$ plane, and the robot's COM projected on that plane can be located as being inside, on the edge, or outside of the foot polygon according to the motion trajectory in the stationary and mobile situations. As shown in Figure 3, the boundary range is defined as the distance between the centroid of the foot polygon (COFP) and $p^{*} . p^{*}$ lies at the point of intersection of the line between the COFP and the COM projected 
on the supporting plane, and the boundary of the polygon. The supporting plane refers to the plane that is formed by the two neighboring feet, $\mathrm{fl}$ and $\mathrm{f} 4$, and the centroid in Figure 3 . If the position of the COM is the same as that of the COFP, the boundary range is assigned as the minimum distance between the COPF and the edge of the foot polygon.

In order to present the balance margin during walking, we first check the point of intersection of $p^{*}\left(x^{*}, y^{*}\right)$ for the boundary range based on the previous research [7]. We used the following procedure to effectively determine the point of intersection. First, if the coordinates of the COM are different from that of the COFP, the $x$ - and $y$-directional positions of the point of intersection can be determined as follows:

$$
\begin{gathered}
x^{*}=\left\{\begin{array}{lll}
\left(b_{12}-b_{c c}\right) /\left(a_{c c}-a_{12}\right) & : & \alpha_{1} \leq \alpha<\alpha_{2} \\
\left(b_{23}-b_{c c}\right) /\left(a_{c c}-a_{23}\right) & : & \alpha_{2} \leq \alpha<\alpha_{3} \\
\left(b_{34}-b_{c c}\right) /\left(a_{c c}-a_{34}\right) & : & \alpha_{3} \leq \alpha<\alpha_{4} \\
\left(b_{41}-b_{c c}\right) /\left(a_{c c}-a_{41}\right) & : & \alpha_{4} \leq \alpha<\alpha_{1}
\end{array}\right. \\
y^{*}=a_{c c} x^{*}+b_{c c}
\end{gathered}
$$

where $a_{i j}(i=1,2,3,4, j=2,3,4,1)$ and $b_{i j}(i=1,2,3,4$, $j=2,3,4,1)$ denote the slope of the line connecting the robotic feet $\mathrm{f} i$ and $\mathrm{f} j$, and the $y$-intercept, respectively. The parameter $a_{c c}$ denotes the slope of the line projecting from the COFP to the $\mathrm{COM}$, and $b_{c c}$ denotes the $y$-intercept. The angle $\alpha$ indicates the angle between the horizontal axis and the projection line, and it can physically be considered as the body's potential direction of fall during walking.

Second, if the $x$-directional positions of the COM and the COFP are identical but their $y$-directional positions are different, the position of the point of intersection can be determined as follows:

$$
\begin{gathered}
x^{*}=x_{\mathrm{COM}} \\
y^{*}=\left\{\begin{array}{lll}
a_{12} x^{*}+b_{12} & : & \alpha_{1} \leq \alpha<\alpha_{2} \\
a_{23} x^{*}+b_{23} & : & \alpha_{2} \leq \alpha<\alpha_{3} \\
a_{34} x^{*}+b_{34} & : & \alpha_{3} \leq \alpha<\alpha_{4} \\
a_{41} x^{*}+b_{41} & : & \alpha_{4} \leq \alpha<\alpha_{1}
\end{array}\right.
\end{gathered}
$$

where $x_{\mathrm{COM}}$ is the $x$-coordinate of the COM.

Third, if the $x$-directional positions of the COM and the COFP are different but their $y$-directional positions are identical, the position of the point of intersection can be determined as follows:

$$
y^{*}=y_{\mathrm{COM}}
$$

$$
x^{*}=\left\{\begin{array}{lll}
\left(y^{*}-b_{12}\right) / a_{12} & : & \alpha_{1} \leq \alpha<\alpha_{2} \\
\left(y^{*}-b_{23}\right) / a_{23} & : & \alpha_{2} \leq \alpha<\alpha_{3} \\
\left(y^{*}-b_{34}\right) / a_{34} & : & \alpha_{3} \leq \alpha<\alpha_{4} \\
\left(y^{*}-b_{41}\right) / a_{41} & : & \alpha_{4} \leq \alpha<\alpha_{1}
\end{array}\right.
$$

where $y_{\mathrm{COM}}$ is the $y$-coordinate of the COM.

Finally, if all positions of the COM and the COFP are identical, the intersection point is determined to be on the boundary of the foot polygon such that the distance between the COFP and the edge of the polygon is at its minimum.

Following the above-mentioned procedure, the $x$ - and $y$ directional boundary ranges, $B_{x}$ and $B_{y}$, can be expressed as follows:

$$
\begin{aligned}
& B_{x}=x^{*}-x_{\mathrm{COFP}} \\
& B_{y}=y^{*}-y_{\mathrm{COFP}} .
\end{aligned}
$$

Given that the boundary ranges include the directional information of walking motion, they are useful for analyzing the directional balance of the quadruped robot in the stationary and walking situations.

We finally define the $x$ - and $y$-directional balance margins, $M_{x}$ and $M_{y}$, as a useful performance measure for identifying quadruped balance as follows:

$$
\begin{aligned}
& M_{x}=B_{x}-x_{\mathrm{COM}} \\
& M_{y}=B_{y}-y_{\mathrm{COM}}
\end{aligned}
$$

where the values of $M_{x}$ and $M_{y}$ denote the difference between each directional boundary range and the current COM position. Therefore, regardless of the sign of the boundary range (positive or negative), we can conclude that the system balance of the robot is within the stable range so long as the sign of the margin is the same as that of the boundary range.

\section{Simulation for Balance Analysis}

This section shows exemplary simulation results to analyze the balance of the quadruped robot system doing some stationary motions or walking.

\subsection{Balance Trend as Stationary Motions}

The effort to identify the system's balance is basically important to achieve the performance of a walking robot system performing a stationary motion or a mobile manipulation. So, the goal of the first simulation is to analyze the stationary balance of 
the robot system by estimating the boundary range and the balance margin described in Section 3. through some exemplary motions.

The three trajectories shown in Figure 4 have been considered for the first simulation. These trajectories imply the body motions for some manipulation tasks of the quadruped robot and they have been specified as the trajectories of the COM projected on the planar ground space. Those COM trajectories are represented by

$$
\begin{aligned}
& x_{\mathrm{COM}}=c_{x}+r_{x} \cos \left(2 \pi t / t_{f}\right) \\
& y_{\mathrm{COM}}=c_{y}+r_{y} \sin \left(2 \pi t / t_{f}\right)
\end{aligned}
$$

where $c_{x}$ and $c_{y}$ represent the $x$ - and $y$-directional center positions of the trajectory, and they have been assigned as 0.65 and 0.70 , respectively. The parameters of $r_{x}$ and $r_{y}$ represent the $x$ - and $y$-directional radius of the trajectory, respectively, and they have been specified in Table 1 for the case studies. Also, the circular time for the trajectories $t_{f}$ has been set as $2.0 \mathrm{~s}$.

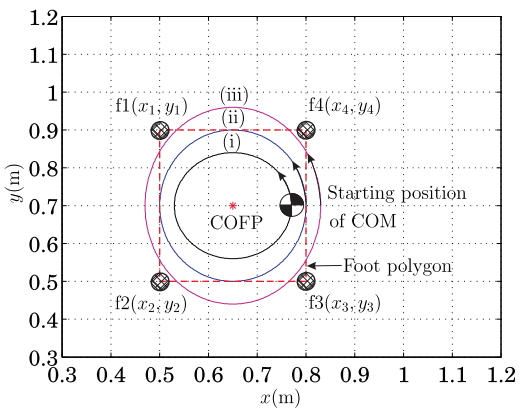

Figure 4. Stationary motion trajectories of the quadruped robot: (i) Case 1, (ii) Case 2, and (iii) Case 3.

Table 1. Parameters of $r_{x}$ and $r_{y}$ for the case studies

\begin{tabular}{llll}
\hline Case & $r_{x}$ & $r_{y}$ & Remarks \\
\hline 1 & 0.12 & 0.14 & Figure 4(i) \\
2 & 0.15 & 0.20 & Figure 4(ii) \\
3 & 0.18 & 0.26 & Figure 4(iii) \\
\hline
\end{tabular}

Table 2. Positions of four feet standing on planar ground

\begin{tabular}{lll}
\hline Foot & $x$ position & $y$ position \\
\hline $\mathrm{f} 1$ & 0.5 & 0.9 \\
$\mathrm{f} 2$ & 0.5 & 0.5 \\
$\mathrm{f} 3$ & 0.8 & 0.5 \\
\hline
\end{tabular}

In particular, the first case in Figure 4 implies that the stationary motion of the robot's body is performed in the inside of the foot polygon and its pattern is an elliptical shape. The second case shows that the motion approaches to the boundary of the foot polygon. The third case shows that some parts of the motion are performed in the outside of the foot polygon. The current positions of the four feet have been specified in Table 2 In this situation, the $x$ - and $y$-directional centroid of the foot polygon, $x_{\mathrm{COFP}}$ and $y_{\mathrm{COFP}}$, can be calculated as $0.65 \mathrm{~m}$ and $0.7 \mathrm{~m}$, respectively [11].

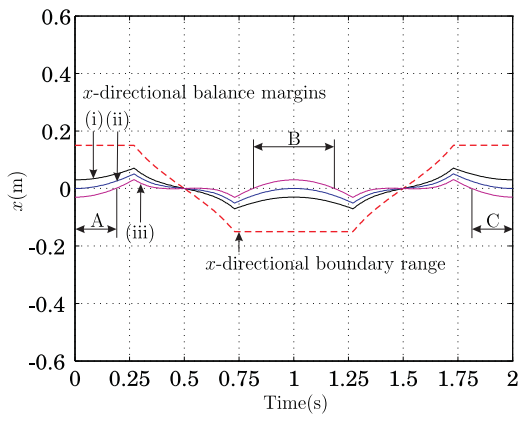

(a) $x$-direction

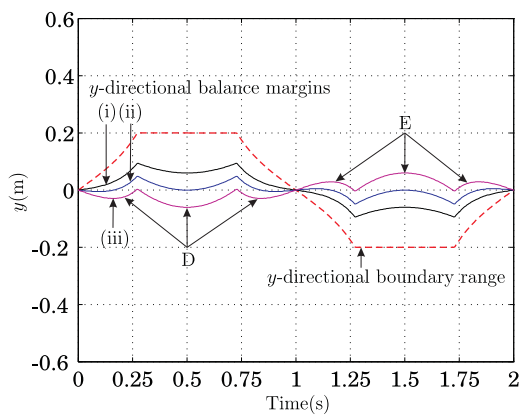

(b) $y$-direction

Figure 5. Boundary ranges and balance margins for the stationary balance: (i) Case 1, (ii) Case 2, and (iii) Case 3.

Figure 5 shows the $x$ - and $y$-directional boundary ranges and margins checked by (7)-(10). If the sign of the margin is reverse compared with that of the corresponding boundary range, the motion can be unstable and then the balance of the walking robot is eventually collapsed in the ranges of A-E pointed in the third case of Figure 5. It is natural because those parts of the motion in the third case are performed in the outside of the foot polygon. In practice, a foot stepping is considerable in order to recover the system balance. In the next section, we discuss on the system balance compensated by the fundamental foot stepping.

\subsection{Balance Compensation by Foot Stepping}

In order to consider the issue of balance compensation by foot stepping, we especially used the two trajectories shown in Figure 6. Actually, the second trajectory is to consider a moving situation for a walk so that some parts of the body's motion 
are out of the stable range of the foot polygon and the walking system is to be unbalanced.

Figure 7 shows the boundary ranges and margins for the given motions in Figure 6 . It is found that the quadruped robot has some margin for the stability in the motion of the first case. However, the balance margins at the regions of A-D in Figure 7 are inadequate to achieve the second motion stably without walking.

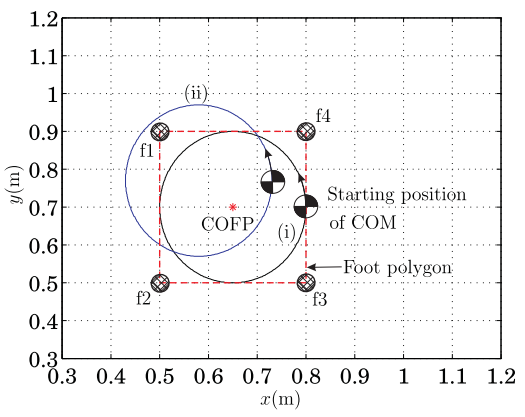

Figure 6. Test motions to identify the system balance in a moving situation for a walk: (i) Case 1 and (ii) Case 2. COM, center of mass; COFP, centroid of the foot polygon.

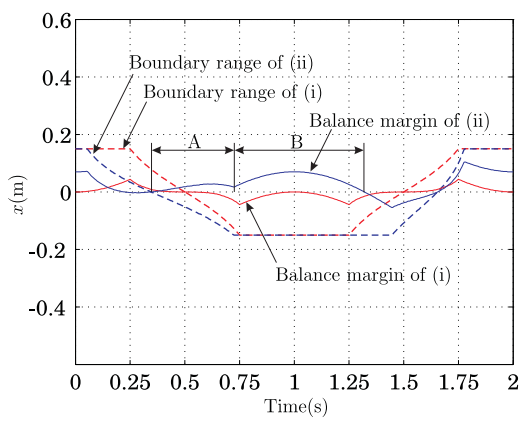

(a) $x$-direction

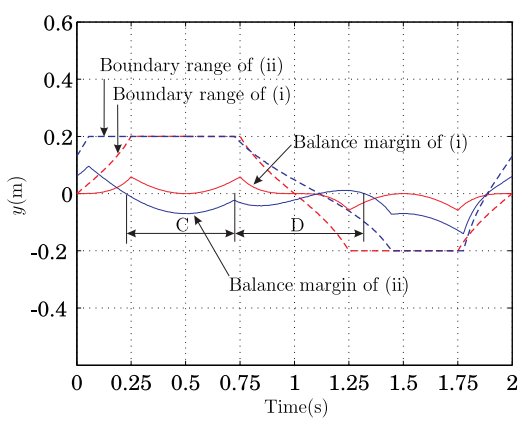

(b) $y$-direction

Figure 7. Boundary ranges and balance margins for the given motions: (i) Case 1 and (ii) Case 2.

Now, we tried to move a foot in order to compensate the unbalance. Figure 8 shows that the first foot has been moved for the balance compensation during the second motion. The objective of this simulation is to show the improvement of the system balance when the first foot $\mathrm{f} 1$ is moving to a suitable position of $x y(0.43,1.05)$. Intuitively, such an effort of foot stepping can contribute to improve the balance margin compared to the second case of Figure 7. That is, the unbalanced regions of $\mathrm{A}$ and $\mathrm{C}$ in Figure 7 can be recovered stably. But it is still unsatisfactory for the robot to follow the trajectory between f1 and $\mathrm{f} 2$.

In order to compensate such an unbalance, the robot can try an additional foot stepping by the second foot $\mathrm{f} 2$ as shown in Figure 9 . Figure 10 shows the resultant boundary ranges and balance margins by the second foot stepping. Finally, we can confirm that the system balance has been made stably. Therefore, our analysis of balance compensation through appropriate foot stepping can be applied for effective gait generation of quadruped robots.

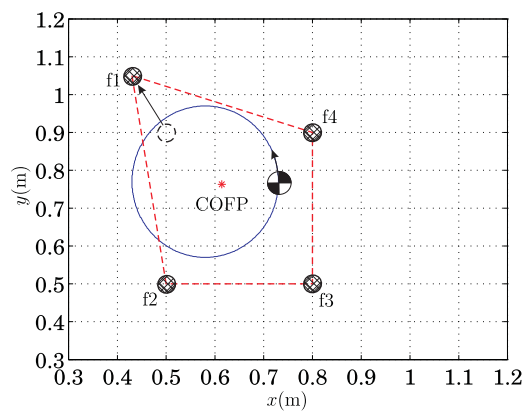

Figure 8. Movement of the first foot for the second motion. COFP, centroid of the foot polygon.

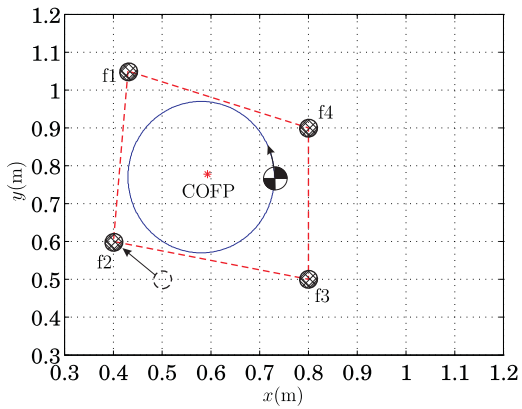

Figure 9. Movement of the second foot for the balance compensation. COFP, centroid of the foot polygon.

\section{Concluding Remarks}

The main conclusion of this study is that the balance of quadruped walking robots can be identified using the proposed boundaryrange-based balance margin. Through exemplary simulations using certain standing and foot-stepping motions, we demonstrated the effectiveness of the proposed measure. Additionally, the effect of balance compensation through appropriate foot stepping was analyzed. We finally concluded that the boundaryrange-based balance margin can be applied to determine the 
foot to be moved and its optimum landing location for effective balance restoration, and that the measure can contribute to improving the system balance over the course of a reasonably long walk. In addition, the proposed measure can be applied to the development of a footstep-planning strategy for ensuring effective quadruped walking.

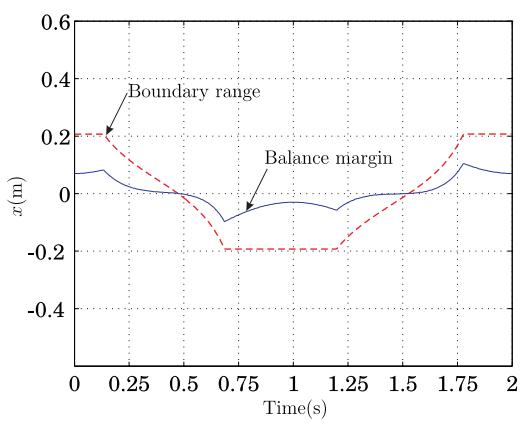

(a) $x$-direction

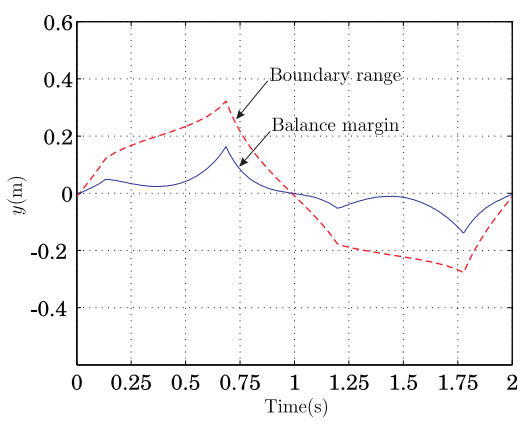

(b) $y$-direction

Figure 10. Boundary ranges and balance margins by second foot stepping.

\section{Acknowledgments}

This research was supported by Kyungsung University Research Grants in 2013.

\section{References}

[1] R. Siegwart and I. R. Nourbakhsh, Introduction to Autonomous Mobile Robots, Cambridge: MIT Press, 2004.

[2] G. S. Hornby, S. Takamura, T. Yamamoto, and M. Fujita, "Autonomous evolution of dynamic gaits with two quadruped robots," IEEE Transactions on Robotics, vol. 21, no. 3, pp. 402-410, Jun. 2005. http://dx.doi.org/10.1109/TRO.2004.839222

[3] Boston Dynamics, "Changing your idea of what robots can do," Available http://www.bostondynamics.com
[4] R. B. Mcghee and G. Iswandhi, "Adaptive locomotion for a multilegged robot over rough terrain," IEEE Transactions on Systems, Man, and Cybernetics, vol. 9, no. 4, pp. 176-182, Apr. 1979. http://dx.doi.org/10.1109/TSMC.1979.4310180

[5] C. Zang and S. Song, "Stability analysis of wave-crab gaits of a quadruped," Journal of Robotic Systems, vol. 7, no. 2, pp. 243-276, Apr. 1990.

[6] B. H. Kim, "Performance index-based evaluation of quadruped robotic walking configuration," International Journal of Fuzzy Logic and Intelligent Systems, vol. 10, no. 4, pp. 308-313, Dec. 2010. http://dx.doi.org/10.5391/IJFIS.2010.10.4.308

[7] B. H. Kim, "Analysis on boundary condition for standing balance of four-legged robots," Journal of Korean Institute of Intelligent Systems, vol. 21, no. 6, pp. 673-678, Dec. 2011. http://dx.doi.org/JKIIS.2011.21.6.673

[8] D. A. Messuri and C. A. Klein, "Automatic body regulation for maintaining stability of a legged vehicle during rough-terrain locomotion," IEEE Journal of Robotics and Automation, vol. RA-1, no. 3, pp. 132-141, Sep. 1985. http://dx.doi.org/10.1109/JRA.1985.1087012

[9] E. Garcia and P. Gonzalez de Santos, "An improved energy stability margin for walking machines subject to dynamic effects," Robotica, vol. 23, no. 1, pp. 13-20, Jan. 2005. http://dx.doi.org/10.1017/S0263574704000487

[10] T. Bretl and S. Lall, "Testing static equilibrium for legged robots," IEEE Transactions on Robotics, vol. 24, no. 4, pp. 794-807, Aug. 2008. http://dx.doi.org/10.1109/TRO.2008.2001360

[11] B. H. Kim, "Analysis on effective walking pattern for multi-legged robots," Journal of Korean Institute of Intelligent Systems, vol. 19, no. 5, pp. 622-628, Oct. 2009. http://dx.doi.org/10.5391/JKIIS.2009.19.5.622

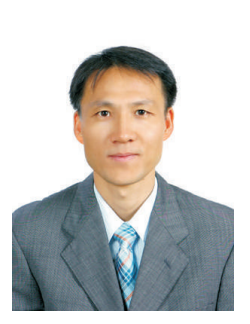

Byoung-Ho Kim received a Ph.D. from the Dept. of Electronics Engineering, Hanyang University, in 2001. He is currently with the Dept. of Mechatronics Engineering, Kyungsung University, Busan, Korea, as an Associate Professor. His research interests include intelligent mobile manipulation, biomimetic system modeling, multi-fingered hands, humanoid robots, and control for advanced robotic applications. 